PO-0192 WITHDRAWN

\section{PO-0193 WITHDRAWN}

\section{PO-0194 THE CLINICAL IMPACT OF POSITIVE BLOOD CULTURE RESULTS IN A DISTRICT GENERAL PAEDIATRIC UNIT}

SE Lawson, PD Donnelly, C Mark, NP Corrigan. Paediatrics, Altnagelvin Hospital, Londonderry, UK

\subsection{6/archdischild-2014-307384.851}

Background and aims Blood cultures are taken to detect bacteraemia and direct antimicrobial therapy. Uncertainty regarding contamination versus bacteraemia leads to financial and human cost through increased investigations and prolonged antimicrobial therapy ${ }^{1}$. Contamination may be due to inadequate aseptic technique, with rates up to $10 \%{ }^{2}$.

This audit aims to establish bacteraemia rates, contamination rates and review if positive blood cultures change clinical management.

Methods A retrospective list of all paediatric blood cultures collected between 7/8/13-7/12/13 was compiled and case-notes of positive results reviewed using a designated proforma.

Contamination was suspected with multiple organisms, particularly if skin flora, exclusively aerobic/anaerobic isolates and reassuring inflammatory markers or clinical condition.

Results

339 blood cultures were taken, 19 were positive.

$47 \%$ of positive results occurred in patients $<1$ year old.

The main indication was pyrexia (63\%)

$42 \%$ of cultures were collected on the ward

Documented asceptic non-touch technique in 1 case $(5 \%)$

Antibiotics were administered before collection in $10 \%$

$68 \%$ of positive results were felt to be contaminants

Our bacteraemia rate is calculated as $1.76 \%$.

$37 \%$ of all positive cultures were repeated including three of six confirmed bacteraeimia cases.

A positive culture result altered clinical management in 32\%.

Conclusions Bacteraemia rates are low at $1.76 \%$. However, there is still a significant contamination rate of $68 \%$, especially in infants where asceptic procedures are difficult. Our results confirm that a blood culture result in isolation is of limited value and clinical correlation is paramount.

\section{REFERENCES}

1 Bates DW, Goldman L, Lee TH. Contaminant blood cultures and resource utilisation. The true consequences of false positive results. JAMA 1991 265:365-369

2 Taking Blood Cultures - a summary of best practice. Saving Lives: reducing infection, delivering clean and safe care. Department of Health, London 2007. Accessed (14th January 2012) via Department of Health Website at: http://hcai. dh.gov.uk/files/2011/03/Document_Blood_culture_FINAL_100826.pdf

\section{PO-0195 A PROSPECTIVE STUDY OF DENGUE FEVER PRESENTATION IN A CHILD COHORT}

${ }^{1} \mathrm{~J}$ Liao, ${ }^{2} \mathrm{~L}$ Foo, ${ }^{3}$ E Hennessy. ${ }^{1}$ Paediatrics, Aberdeen Royal Infirmary, Aberdeen, UK; ${ }^{2}$ Sungai Buloh Hospital, Chidrlen's Ward, Kuala Lumpur, Malaysia; 'Paediatrics, Aberdeen Royal Infirmary, Aberdeen, UK

\subsection{6/archdischild-2014-307384.852}

Background Dengue fever (DF) infects between 50 and 100 million people each year. Local DF transmission was first reported in Europe in 2010 and WHO now warns of a possible DF outbreak in Europe.

DF is usually self-limiting in the population (overall mortality rate of $<1 \%$.) However, $90 \%$ of patients with dengue haemorrhagic fever (severe dengue) are under 15 years old. The initial presentation of both is similar in children. Mortality rate in severe dengue is $2.5 \%$.

Aim To describe DF presentation in a child cohort

Method During an 8 week period, data were collected for 19 children diagnosed with DF and included demography, previous dengue infection, clinical presentation and time interval between symptom onset and admission.

Results All children with DF were under 15 years old. None had previous episodes, complications or progression to dengue haemorrhagic fever. $74 \%$ of children were living in red flagged areas when symptoms occurred. Close contacts with a recent DF diagnosis were identified in $37 \%$. Pyrexia was a first symptom in $84 \%$. Other symptoms included rashes (42\%), cough (37\%), loss of appetite (37\%), vomiting (37\%), dizziness (37\%) and headache (37\%), as well as 14 other symptoms of lower frequency. An average of 5 days separated initial symptoms and admission.

Conclusion In considering differential diagnosis in children presenting in Europe with non-specific symptoms such as those found in this study, the possibility of DF should not be omitted. A travel and contact history is even more important if this potentially serious infection is to be recognised.

\section{PO-0196 EVOLUTION OF INFECTIONS IN FOUR CACHECTIC CHILDREN. CASE REPORTS}

L Marinau, S Raciula. Pediatric: Mother and Child, University of Medicine and Pharmacy of Craiova, Craiova, Romania

\subsection{6/archdischild-2014-307384.853}

We can diagnose cachexy in children whose BMI is $<5$ percentiles and under 3-rd negative deviation. The aetiology of poor state of nutrition can be: incorrect feeding, frequent infections, congenital defects, improper care or a combination of the previous situations.

Authors present four cachectic patients: two children suffering of trisomy 21, who had associated each one heart malformation: first, a boy, N. S., aged 7 and weighting $12 \mathrm{Kg}$ had Fallot tetralogy and bacterial endocarditis; second, a girl, P. S, 43 months aged, weighting $10 \mathrm{Kg}$, had ventricular septal defect and acute interstitial pneumonia. The third cachectic patient was a girl aged 8, weighting $13 \mathrm{~kg}$, D. C. with Seckel syndrome, admitted for staphylococcal pneumonia; the fourth is a 11 years girl weighting $12 \mathrm{Kg}$, P. D. with spastic tetraparesis, microcefaly, diagnosed with severe sepsis.

Only the boy suffering of endocarditis, evaluated to MODS and death; the three girls were discharged healed. Trisomy 21 caused immunodeficiency and cachexy also permitted severe infections. The boy had severe hypoxia, but girls had a normal oxygen saturation. The aetiology of poor state of nutrition was combinated: congenital defects, improper diet, frequent infections, inadequate care (three patients lived in orphanage, a girl had only mother). Pneumonia is frequent in thin children, with birth malformations. Congenital heart defects could been complicated to endocarditis.

Cachexy in malformed children is an important cause of immunodeficiency which leads to severe, sometimes letal infections. Infections produce denutrition and cachexy promotes severe sepsis, especially in parentless children. 


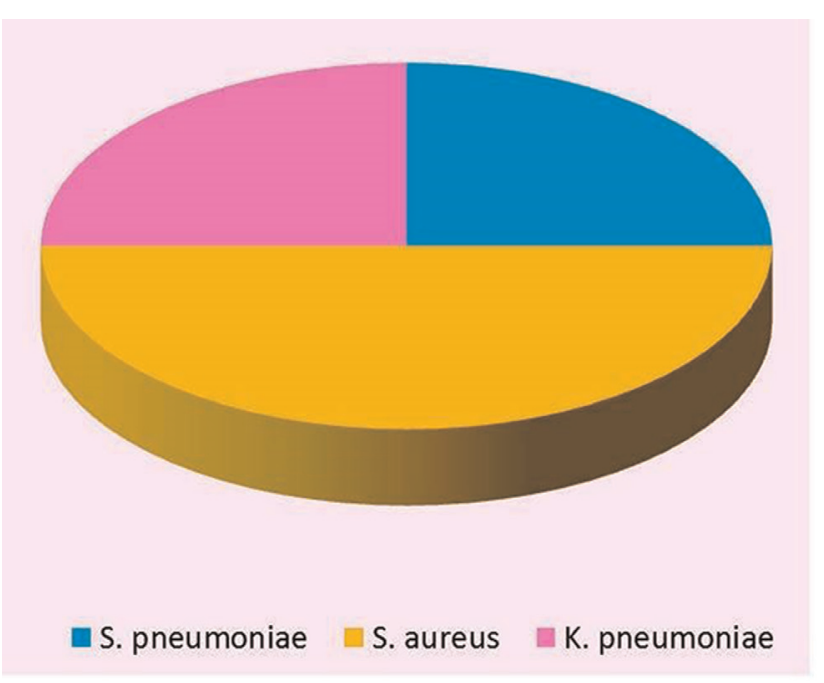

Blood culture $(n=4)$

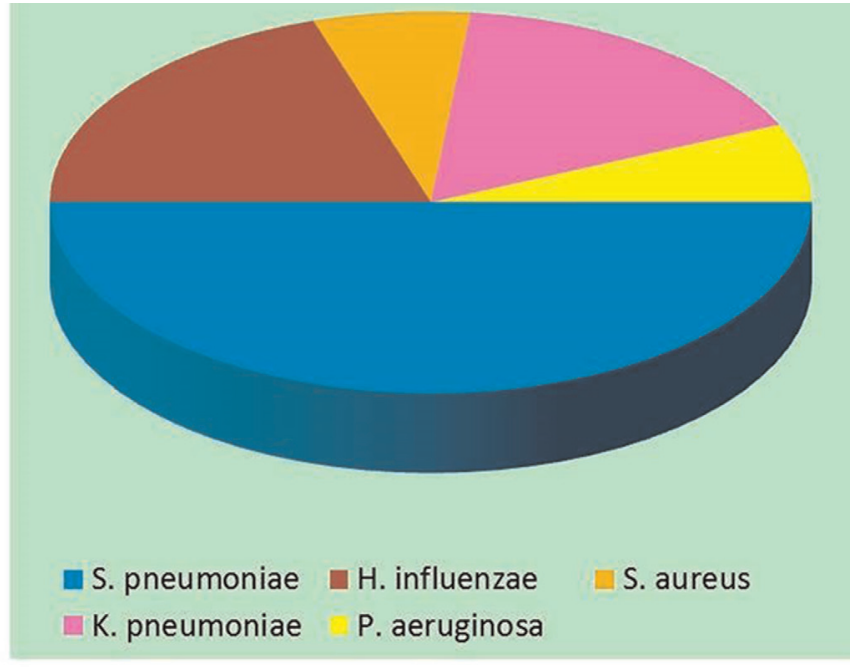

NPA culture $(\mathbf{n}=\mathbf{3 0})$

Abstract P0-0197 Figure 1 Bacteria detected by culture (Blood and NPA)

\section{PO-0197 BACTERIAL CULTURE VERSUS PCR FOR ETIOLOGIC DIAGNOSIS OF COMMUNITY ACQUIRED PNEUMONIA- RESULTS FROM CAPES (COMMUNITY ACQUIRED PNEUMONIA ETIOLOGY STUDY)}

1JL Mathew, 'S Singhi, ${ }^{2} \mathrm{P}$ Ray, ${ }^{3} \mathrm{BV}$ Ravi Kumar, ${ }^{1} \mathrm{~A}$ Bansal, ${ }^{4} \mathrm{~A}$ Nilsson. ${ }^{1}$ Advanced Pediatrics Centre, Post Graduate Institute of Medical Education and Research, Chandigarh, India; ${ }^{2}$ Medical Microbiology, Post Graduate Institute of Medical Education and Research, Chandigarh, India; ${ }^{3}$ Managing Director, Xcyton Diagnostics Pvt Ltd, Bangalore, India; ${ }^{4}$ Astrid Lindgren Children's Hospital, Karolinska University Hospital, Stockholm, Sweden

\subsection{6/archdischild-2014-307384.854}

Background PCR technologyholds promise to overcome the limitations of bacterial culture for confirming bacterial aetiology in childhood pneumonia.

Aims To compare the diagnostic yield using bacterial culture versus PCR in a cohort of children with community acquired pneumonia (CAP).

Methods CAPES is single-centre cohort study of 2000 consecutively enrolled children (1 month-12 years) with CAP(World Health Organisation definition). All underwent blood and

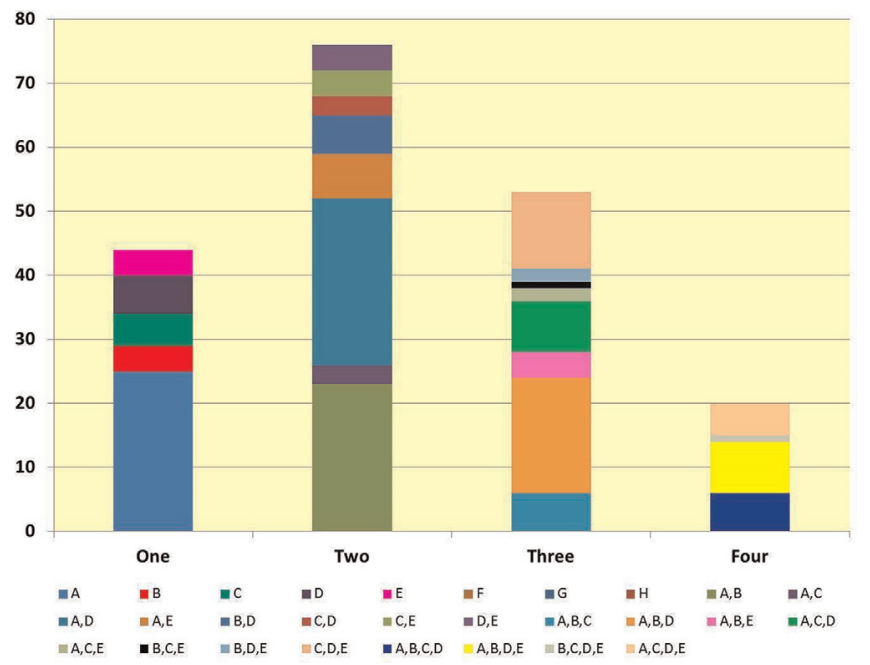

Abstract P0-0197 Figure 2 Bacteria detected by multiplex PCR nasopharyngeal aspirate (NPA) culture for bacteria. In addition, a randomly selected sub-group $(10 \%, \mathrm{n}=200)$ underwent multiplex PCR on NPA samples to identify eight pathogenic bacteria. Results In the sub-cohort of 200 children, pathogenic organisms were isolated from blood and NPA in 4(2\%) and $30(15 \%)$ respectively (Figure 1). The main cohort showed similar proportions $(1.7 \%$ and $14.0 \%)$ and distribution of organisms, suggesting absence of selection bias. Multiplex PCR(Figure 2) yielded traces of bacteria in 193(96.5\%), with one organism detected in $44(22 \%)$, two in $76(38 \%)$, three in $53(26.5 \%)$, and four in 20 (10\%). Etiologic diagnosis could be confirmed by bacterial culture in $4.5 \%$ cases, whereas PCR confirmed in $22 \%$.

Conclusion Bacterial culture techniques appear to have limited sensitivity for etiologic diagnosis, whereas PCR has much higher sensitivity, although detection of multiple pathogenic bacteria precluded confirmation of aetiology in the majority.

\section{PO-0198 FERRITIN AND SERUM IRON IN PAEDIATRIC FEBRILE ILLNESS}

${ }^{1}$ A Nasir, ${ }^{2}$ S James, ${ }^{3}$ D Perry, ${ }^{4}$ J Meza. ${ }^{1}$ Pediatrics, University of Nebraska Medical Center, Omaha, USA; ${ }^{2}$ Emergency, Children's Hospital and Medical Center, Omaha, USA; ${ }^{3}$ Pathology, Children's Hospital and Medical Center, Omaha, USA; ${ }^{4}$ Statistics, University of Nebraska Medical Center, Omaha, USA

\subsection{6/archdischild-2014-307384.855}

Introduction Ferritin is the major intracellular iron binding protein in the body. Serum ferritin is an inflammatory marker. Iron sequestration is one of the innate immune responses to infection. The goal of this research was to investigate the role of serum ferritin and serum iron as clinically useful markers of infection in the paediatric emergency department.

Methods Multiple inflammatory markers, including C-reactive protein, procalcitonin, and serum ferritin, and other iron studies were measured in 37 children, from 3 months through 8 years of age, presenting to the emergency department with temperature of $\geq 39$ degrees Celsius, and 38 patients in the same age group with non-febrile illness (controls). Patients with chronic inflammatory or rheumatologic conditions and those with renal or hepatic failure were excluded. 